\title{
Disentangling the genetics of sarcopenia: prioritization of NUDT3 and KLF5 as genes for lean mass \& HLA-DQB1-AS1 for hand grip strength with the associated enhancing SNPs \& a scoring system
}

\author{
Abhishek Narain Singh ${ }^{1,2^{*}}$ and Bili Gasman ${ }^{2}$
}

\begin{abstract}
Background: Sarcopenia is a skeletal muscle disease of clinical importance that occurs commonly in old age and in various disease sub-categories. Widening the scope of knowledge of the genetics of muscle mass and strength is important because it may allow to identify patients with an increased risk to develop a specific musculoskeletal disease or condition such as sarcopenia based on genetic markers.

Methods: We used bioinformatics tools to identify gene loci responsible for regulating muscle strength and lean mass, which can then be a target for downstream lab experimentation validation. Single nuclear polymorphisms (SNPs) associated with various disease traits of muscles and specific genes were chosen according to their muscle phenotype association p-value, as traditionally done in Genome Wide Association Studies, GWAS. We've developed and applied a combination of expression quantitative trait loci (eQTLs) and GWAS summary information, to prioritize causative SNP and point out the unique genes associated in the tissues of interest (muscle).

Results: We found NUDT3 and KLF5 for lean mass and HLA-DQB1-AS1 for hand grip strength as candidate genes to target for these phenotypes. The associated regulatory SNPs are rs464553, rs 1028883 and rs3129753 respectively.

Conclusion: Transcriptome Wide Association Studies, TWAS, approaches of combining GWAS and eQTL summary statistics proved helpful in statistically prioritizing genes and their associated SNPs for the disease phenotype of study, in this case, Sarcopenia. Potentially regulatory SNPs associated with these genes, and the genes further prioritized by a scoring system, can be then wet lab verified, depending on the phenotype it is hypothesized to affect.
\end{abstract}

Keywords: SNPS, GWAS, eQTL, TADs, TWAS, muscle, SMR, sarcopenia

\footnotetext{
* Correspondence: abhishek.narain@iitdalumni.com

'A.I. Virtanen Institute for Molecular Sciences, University of Eastern Finland, Kuopio, Finland

${ }^{2}$ Faculty of Medicine, Bar Ilan University, Safed, Israel
}

(c) The Author(s). 2020 Open Access This article is licensed under a Creative Commons Attribution 4.0 International License, which permits use, sharing, adaptation, distribution and reproduction in any medium or format, as long as you give appropriate credit to the original author(s) and the source, provide a link to the Creative Commons licence, and indicate if changes were made. The images or other third party material in this article are included in the article's Creative Commons licence, unless indicated otherwise in a credit line to the material. If material is not included in the article's Creative Commons licence and your intended use is not permitted by statutory regulation or exceeds the permitted use, you will need to obtain permission directly from the copyright holder. To view a copy of this licence, visit http://creativecommons.org/licenses/by/4.0/. The Creative Commons Public Domain Dedication waiver (http://creativecommons.org/publicdomain/zero/1.0/) applies to the data made available in this article, unless otherwise stated in a credit line to the data. 


\section{Background}

Many diseases known to man originate from more than one genetic locus. Sarcopenia for example is multifactorial [1] degenerative loss of skeletal muscle mass, a condition that might pose a great risk for the aging world population. Since 2006, GWAS have allowed us to trace the multiple genetic factors for various traits using statistical tools that can lead to a more effective research of specific loci of interest [2]. The data produced by these studies, which now rank in the thousands, is available online so further downstream research can be conducted, and new results can be incorporated. This is indeed valuable, since musculoskeletal diseases are one of the leading causes of disability in the world [3]; treatment of these diseases costs the world medical industry around 125 billion dollars annually [3].

In this paper, we present the combination of summary level data from GWAS and publicly available eQTLs such as those from studies by GTEx [4] and Westra et al. [5]. Based on available data and our approach of combining phenotype-associated SNPs (Single Nucleotide Polymorphism) and tissue-relevant gene-associated SNPs TADs (topologically associated domains) were plotted at the regions of interest. A TAD is a self-interacting genomic region, meaning that DNA sequences within a TAD physically interact with each other more frequently than with sequences outside the TAD [6].

We developed and applied a combination of expression quantitative trait loci (eQTLs) and GWAS summary information, to prioritize causative SNP and point out the unique genes associated in the tissues of interest (muscle).

\section{Method}

The results of GWAS for lean mass (LM) and hand grip strength (HG) were published in studies by Karasik et al. [7], Zilikens et al. [8], Willems et al. [9] and Tikkanen et al. [10], in various large human populations. According to consensus in the literature, we used the genome-wide significance threshold of $5^{*} 10^{-8}$ to consider SNPs to be associated for a follow-up. The summary of eQTL data was obtained by studies by Westra et al. [5] and by the GTEx [4] consortium. From the study of Westra et al. eQTLs of HSMM (Human skeletal muscle myoblasts) culture were obtains, while the GTEx consortium EQTLs were from human striated muscle samples. With these data sets, we executed "Summary-data-based Mendelian randomization" (SMR) analysis using the method as proposed by Zhu et al. [11] and utilizing the "SMR tool" program, version 0.710. We chose not to investigate the SNPs for further categorization of pleiotropy or causality, which can be done using the Heidi test [11]. For the case of GTEx eQTL summary data, the execution was done for all tissues, and then we observed for genes which were enriched specifically in skeletal muscle tissue or specifically compared to the aggregate of all other tissue types. For the genes of interest as described in the above method, we went on to plot and examine TADs at the relevant regions in corresponding skeletal muscle tissues such as the psoas (striated) and bladder (smooth muscle) as done by Schmitt et al. [12]

\section{Results}

GTEx tissue analysis found that for lean mass, two genes: NUDT3 and KLF5, were enriched in skeletal muscles (Figs. 1, 2), and they were also found in

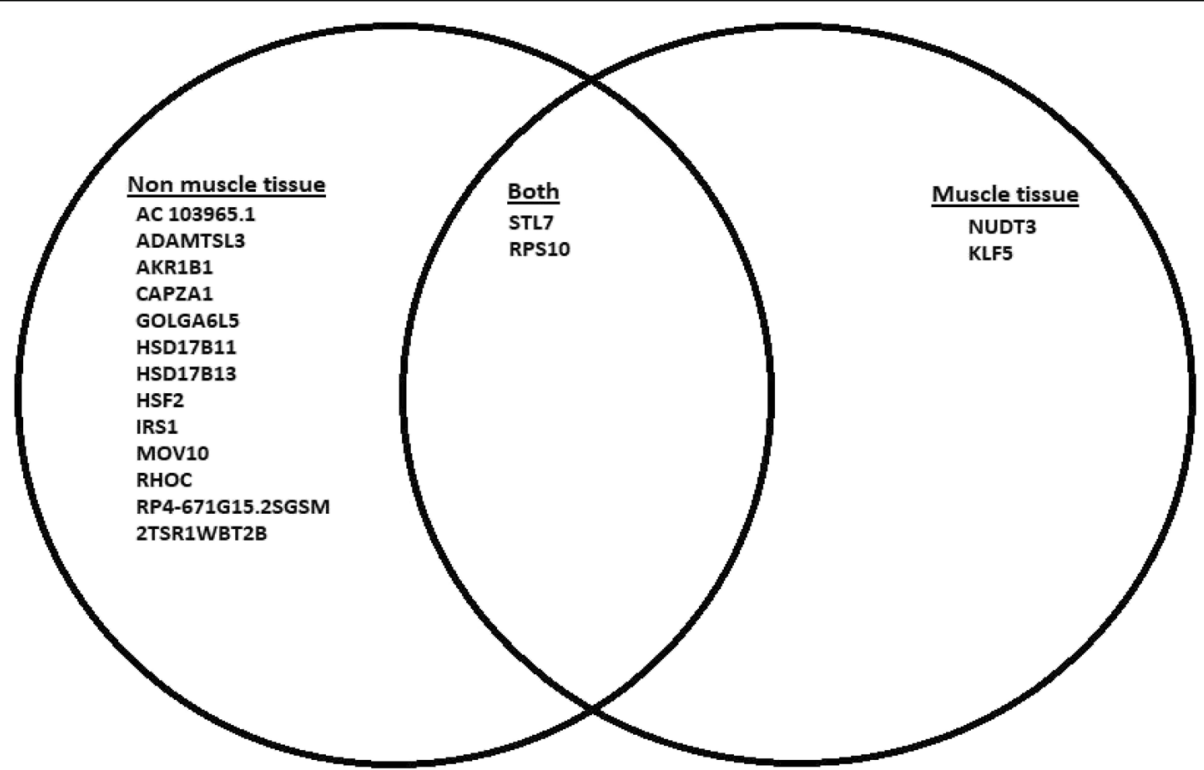

Fig. 1 Gene expression based on SMR of GTEx eQTLs and whole-body lean mass GWAS 


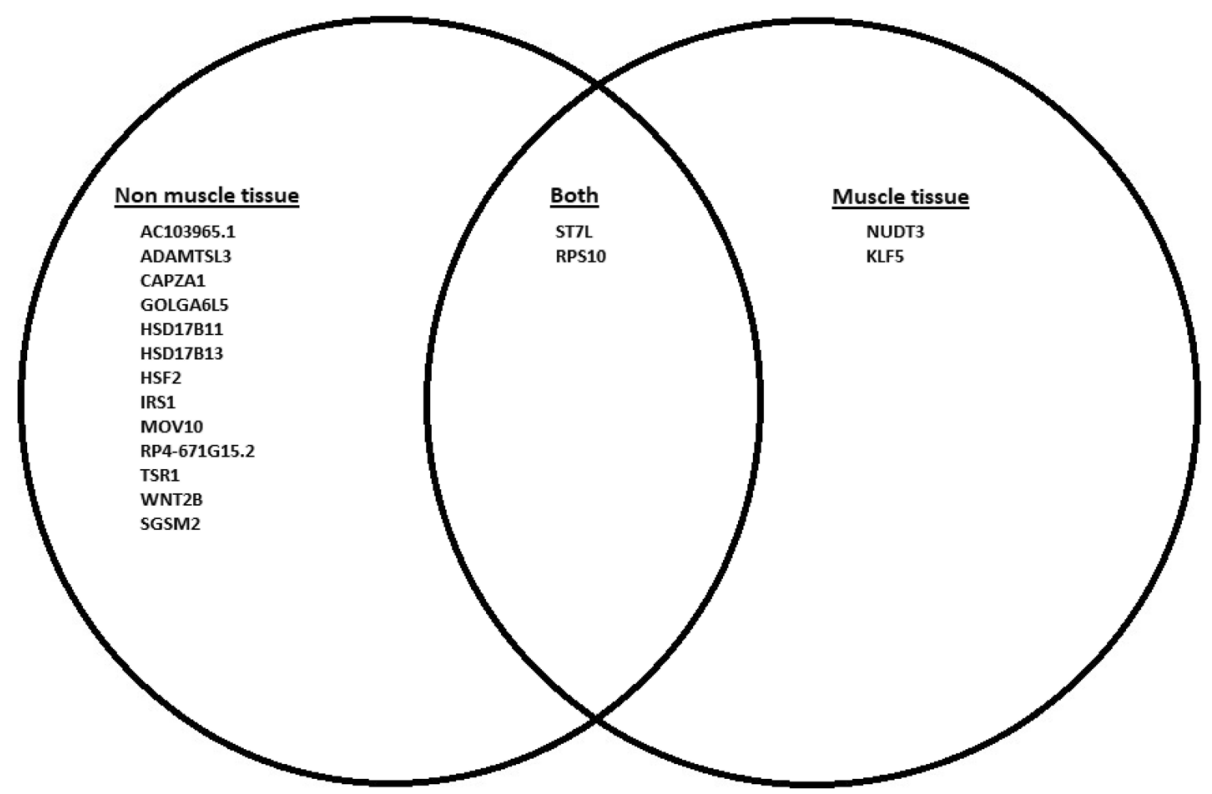

Fig. 2 Gene expression based on SMR of GTEx eQTLS and appendicular lean mass GWAS

Westra et al. [5] eQTL analysis for appendicular lean mass (Table 1), although not in whole body in the case of Westra et al. [5] study (Table 2). Venn diagram in Fig. 1 is derived from Tables 3 and 4; Venn diagram in Fig. 2 is derived from Tables 5 and 6. In the GTEx tissue analysis for the hand grip trait, we found one gene, $H L A-D Q B 1-A S 1$, which was specifically enriched in skeletal tissue compared to other tissues (Fig. 3), with the associated SNP as rs3129753. The Venn diagram in Fig. 3 is derived from Tables 7 and 8. Many other genes found to be enriched in skeletal muscle tissues and other tissues in common intersection (Figs. 1, 2 and 3) were also found in Westra eQTL analysis with our GWAS summary dataset. The second priority should be given to the genes found to be enriched in skeletal muscle tissue as well as any other tissue. Clearly, NUDT3 and KLF5 are very strong candidate genes for lean mass study, and their associated regulating SNP are rs464553 and

Table 1 Metal SMR of Westra et al. eQTLs and appendicular lean mass GWAS

\begin{tabular}{lllll}
\hline probe ID & chromosome & Gene & $\begin{array}{l}\text { base pair of } \\
\text { probe }\end{array}$ & $\begin{array}{l}\text { associated } \\
\text { SNP }\end{array}$ \\
\hline ENSG00000007341.14 & 1 & ST7L & $113,122,614$ & rs1110043 \\
ENSG00000272325.1 & 6 & NUDT3 & $34,307,498$ & rs464553 \\
ENSG00000124614.9 & 6 & RPS10 & $34,389,566$ & rs464553 \\
ENSG00000102554.9 & 13 & KLF5 & $73,640,395$ & rs1028883 \\
\hline
\end{tabular}

rs1028883 respectively. TAD plots for the psoas and bladder tissues (which are skeletal and smooth muscle types, respectively) were plotted (Figs. 4, 5, 6 and 7) where KLF5 is seen to be present within a FIRE [12] (frequently interacting region) within the TAD of chromosome 13 in bladder (M.Detrusor) muscle (Fig. 4).

\section{Discussion}

Apart from the combination of GWAS and eQTL summaries for the tissue of interest and searching for exclusivity of gene enhancement and the presence of SNP regulation near TAD boundaries, we also incorporated a novel scoring system to prioritize genes for functional validation of our results. Functional validation is a slow and costly process. Validation can take months and even years to complete without a promise of a positive result, hence a scoring system is vital

Table 2 SMR of Westra et al. eQTLs and whole-body lean mass GWAS

\begin{tabular}{lllll}
\hline probe ID & chromosome & Gene & $\begin{array}{l}\text { base pair of } \\
\text { probe }\end{array}$ & associated SNP \\
\hline ILMN_1659926 & 1 & ST7L & $112,886,029$ & rs1110043 \\
ILMN_2410113 & 1 & ST7L & $112,900,108$ & rs7522288 \\
ILMN_1725700 & 1 & MOV10 & $113,044,669$ & rs2999156 \\
ILMN_1673305 & 1 & RHOC & $113,045,326$ & rs2999156 \\
ILMN_2313730 & 1 & RHOC & $113,045,386$ & rs2999156 \\
ILMN_1701731 & 7 & AKR1B1 & $133,777,993$ & rs7795758 \\
\hline
\end{tabular}


Table 3 SMR of GTEx eQTLs and lean mass GWAS, results for all tissue types

\begin{tabular}{lllll}
\hline probe ID & chromosome & Gene & $\begin{array}{l}\text { base pair } \\
\text { of probe }\end{array}$ & $\begin{array}{l}\text { associated } \\
\text { SNP }\end{array}$ \\
\hline ENSG00000007341.14 & 1 & ST7L & $113,122,614$ & rs1110043 \\
ENSG00000116489.8 & 1 & CAPZA1 & $113,188,330$ & rs1110043 \\
ENSG00000169047.5 & 2 & IRS1 & $227,630,254$ & rs2943656 \\
ENSG00000198189.6 & 4 & HSD17B11 & $88,285,150$ & rs9991501 \\
ENSG00000124614.9 & 6 & RPS10 & $34,389,566$ & rs464553 \\
ENSG00000025156.8 & 6 & HSF2 & $122,737,477$ & rs1991642 \\
ENSG00000007341.14 & 1 & ST7L & $113,122,614$ & rs1110043 \\
ENSG00000007341.14 & 1 & ST7L & $113,122,614$ & rs1110043 \\
ENSG00000007341.14 & 1 & ST7L & $113,122,614$ & rs1110043 \\
ENSG00000007341.14 & 1 & ST7L & $113,122,614$ & rs1110043 \\
ENSG00000007341.14 & 1 & ST7L & $113,122,614$ & rs1110043 \\
ENSG00000116489.8 & 1 & CAPZA1 & $113,188,330$ & rs1110043 \\
ENSG00000170509.7 & 4 & HSD17B13 & $88,234,499$ & rs9991501 \\
ENSG00000198189.6 & 4 & HSD17B11 & $88,285,150$ & rs9991501 \\
\hline
\end{tabular}

for scrutinizing and grading our results to asses which gene might have an effect on muscle health. The process of functional validation is vital for a few reasons. For one, relying on TADs has its limitations. Chromosomes separate active and inactive chromatin into compartments $\mathrm{A}$ and $\mathrm{B}$, respectively where compartment A correlates with high gene expression, active histone marks, and early replication timing, whereas the compartment B replicates late, is enriched with repressive histone modifications and has low gene expression. Compartments can be further subdivided into megabase-sized genomic regions known as topologically associating domains (TADs) $[13,14]$. the function of TADs is not fully understood yet, although disrupting the TADs e.g. because of SNPs or InDels (insertions deletions) may result in the establishment of novel inter-TAD interactions. These have been shown to be associated with misexpression of Hox genes [15], up-regulation of protooncogenes [16], and developmental disorders [14].

Table 4 SMR of GTEx eQTLs and lean mass GWAS, results for skeletal muscles

\begin{tabular}{lllll}
\hline probe ID & chromosome & Gene & $\begin{array}{l}\text { base pair of } \\
\text { probe }\end{array}$ & $\begin{array}{l}\text { associated } \\
\text { SNP }\end{array}$ \\
\hline ENSG00000007341.14 & 1 & ST7L & $113,122,614$ & rs 1110043 \\
ENSG00000272325.1 & 6 & NUDT3 & $34,307,498$ & rs464553 \\
ENSG00000124614.9 & 6 & RPS10 & $34,389,566$ & rs464553 \\
ENSG00000102554.9 & 13 & KLF5 & $73,640,395$ & rs1028883 \\
\hline
\end{tabular}

Table 5 Metal SMR of GTEx eQTLs and appendicular lean mass GWAS

\begin{tabular}{lllll}
\hline probe ID & chromosome & Gene & $\begin{array}{l}\text { base pair } \\
\text { of probe }\end{array}$ & $\begin{array}{l}\text { associated } \\
\text { SNP }\end{array}$ \\
\hline ENSG000000007341.14 & 1 & ST7L & $113,122,614$ & rs 1110043 \\
ENSG00000116489.8 & 1 & CAPZA1 & $113,188,330$ & rs 1110043 \\
ENSG00000169047.5 & 2 & IRS1 & $227,630,254$ & rs 2943656 \\
ENSG00000198189.6 & 4 & HSD17B11 & $88,285,150$ & rs 9991501 \\
ENSG00000124614.9 & 6 & RPS10 & $34,389,566$ & rs 464553 \\
ENSG00000025156.8 & 6 & HSF2 & $122,737,477$ & rs 1991642 \\
ENSG00000007341.14 & 1 & ST7L & $113,122,614$ & rs 1110043 \\
ENSG00000007341.14 & 1 & ST7L & $113,122,614$ & rs 1110043 \\
ENSG00000007341.14 & 1 & ST7L & $113,122,614$ & rs 1110043 \\
ENSG00000007341.14 & 1 & ST7L & $113,122,614$ & rs 1110043 \\
ENSG000000007341.14 & 1 & ST7L & $113,122,614$ & rs 1110043 \\
ENSG00000116489.8 & 1 & CAPZA1 & $113,188,330$ & rs 1110043 \\
ENSG00000170509.7 & 4 & HSD17B13 & $88,234,499$ & rs9991501 \\
ENSG00000198189.6 & 4 & HSD17B11 & $88,285,150$ & rs9991501 \\
ENSG00000007341.14 & 1 & ST7L & $113,122,614$ & rs65377448 \\
ENSG00000273483.1 & 1 & RP4-671G15.2 & $113,060,742$ & rs 1110043 \\
ENSG00000134245.13 & 1 & WNT2B & $113,040,975$ & rs1110043 \\
ENSG00000273483.1 & 1 & RP4-671G15.2 & $113,060,742$ & rs 1110043 \\
ENSG00000007341.14 & 1 & ST7L & $113,122,614$ & rs7522288 \\
\hline & & & &
\end{tabular}

Furthermore, functional validation might also allow us to identify drugs that affect muscle in ways unknown before and therefore to reposition existing drugs to other uses, in accordance to their newly found target such as targeted gene therapy as discussed in context of next generation sequencing in drug development [17]. This serves two purposes, first is validation of the scoring system itself as an algorithm for GWAS result validation, and the more important one is validation of new targets for further research and potentially, repositioning of existing drugs.

Our approach has its limitations and requires validation, as one can observe from the results. In spite the fact that TADs that were plotted by our approach of combining phenotype-associated SNPs and tissuerelevant gene-associated SNPs show that the genes of

Table 6 SMR of GTEx eQTLs and appendicular lean mass GWAS, results for skeletal muscle

\begin{tabular}{lllll}
\hline probe ID & chromosome & Gene & $\begin{array}{l}\text { base pair of } \\
\text { probe }\end{array}$ & $\begin{array}{l}\text { associated } \\
\text { SNP }\end{array}$ \\
\hline ENSG00000007341.14 & 1 & ST7L & $113,122,614$ & rs1110043 \\
ENSG00000272325.1 & 6 & NUDT3 & $34,307,498$ & rs464553 \\
ENSG00000124614.9 & 6 & RPS10 & $34,389,566$ & rs464553 \\
ENSG00000102554.9 & 13 & KLF5 & $73,640,395$ & rs1028883 \\
\hline
\end{tabular}




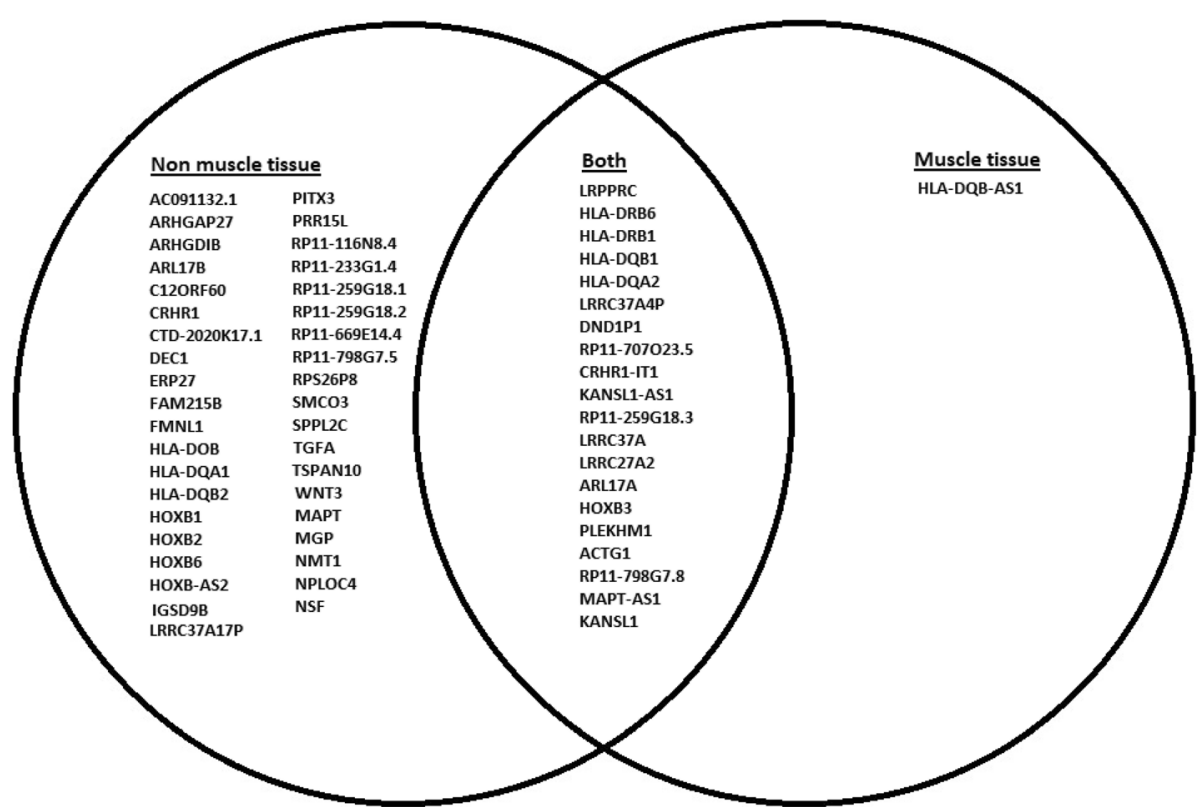

Fig. 3 Gene expression based on Handgrip GWAS data and GTEx eQTL enrichment analysis

interest are located within a frequently interacting region, the rest of the data regarding these genes doesn't support our hypothesis that they in fact have an effect on muscle health. NUDT3 (Nudix Hydrolase 3) for example, codes for the Nudix protein which act as

Table 7 SMR of GTEx skeletal muscle eQTLs and handgrip GWAS

\begin{tabular}{|c|c|c|c|c|}
\hline probe ID & chromosome & Gene & $\begin{array}{l}\text { base pair } \\
\text { of probe }\end{array}$ & $\begin{array}{l}\text { associated } \\
\text { SNP }\end{array}$ \\
\hline ENSG00000138095.14 & 2 & $\angle R P P R C$ & $44,168,395$ & rs6544736 \\
\hline ENSG00000229391.3 & 6 & HLA-DRB6 & $32,524,144$ & rs3129751 \\
\hline ENSG00000196126.6 & 6 & HLA-DRB1 & $32,552,085$ & rs521539 \\
\hline ENSG00000223534.1 & 6 & HLA-DQB1-AS1 & $32,628,319$ & rs3129753 \\
\hline ENSG00000179344.12 & 6 & $H L A-D Q B 1$ & $32,631,702$ & rs521539 \\
\hline ENSG00000237541.3 & 6 & HLA-DQA2 & $32,712,055$ & rs6931277 \\
\hline ENSG00000225190.4 & 17 & PLEKHM1 & $43,540,690$ & rs62055516 \\
\hline ENSG00000214425.2 & 17 & $\angle R R C 37 A 4 P$ & $43,603,193$ & rs389978 \\
\hline ENSG00000266918.1 & 17 & RP11-798G7.8 & $43,610,073$ & rs2668668 \\
\hline ENSG00000264070.1 & 17 & DNDIP1 & $43,663,766$ & rs2266497 \\
\hline ENSG00000263503.1 & 17 & RP11-707023.5 & $43,678,970$ & rs2266497 \\
\hline ENSG00000204650.9 & 17 & CRHR1-IT1 & $43,711,638$ & rs62061802 \\
\hline ENSG00000264589.1 & 17 & MAPT-ASI & $43,946,991$ & rs2668668 \\
\hline ENSG00000120071.8 & 17 & KANSL1 & $44,205,007$ & rs62073098 \\
\hline ENSG00000214401.4 & 17 & KANSL1-AS1 & $44,272,515$ & rs55974014 \\
\hline ENSG00000262539.1 & 17 & RP11-259G18.3 & $44,337,444$ & rs56364632 \\
\hline ENSG00000176681.10 & 17 & $\angle R R C 37 A$ & $44,392,629$ & rs17660294 \\
\hline ENSG00000238083.3 & 17 & LRRC37A2 & $44,609,846$ & rs439558 \\
\hline ENSG00000185829.11 & 17 & ARL17A & $44,625,578$ & rs3865315 \\
\hline ENSG00000120093.7 & 17 & HOXB3 & $46,646,933$ & rs890432 \\
\hline ENSG00000184009.5 & 17 & ACTGI & $79,483,935$ & rs6565586 \\
\hline
\end{tabular}

homeostatic checkpoints at important stages in nucleoside phosphate metabolic pathways, guarding against elevated levels of potentially dangerous intermediates [18]. GWAS associate RSP-NUDT3 readthrough to BMI with a $p$-value of $4 * 10^{-12}$ [19]. The Malacards database also associates NUDT3 with hyperinsulinism and obesity in

Table 8 GTEx Handgrip GWAS data with eQTL aggregated SMR

\begin{tabular}{|c|c|c|c|c|}
\hline probe ID & chromosome & Gene & $\begin{array}{l}\text { base pair } \\
\text { of probe }\end{array}$ & $\begin{array}{l}\text { associated } \\
\text { SNP }\end{array}$ \\
\hline ENSG00000138095.14 & 2 & $\angle R P P R C$ & $44,168,395$ & rs6544736 \\
\hline ENSG00000229391.3 & 6 & HLA-DRB6 & $32,524,144$ & rs3129751 \\
\hline ENSG00000196126.6 & 6 & HLA-DRB1 & $32,552,085$ & rs521539 \\
\hline ENSG00000223534.1 & 6 & HLA-DQB1-AS1 & $32,628,319$ & rs3129753 \\
\hline ENSG00000179344.12 & 6 & $H L A-D Q B 1$ & $32,631,702$ & rs521539 \\
\hline ENSG00000237541.3 & 6 & HLA-DQA2 & $32,712,055$ & rs6931277 \\
\hline ENSG00000225190.4 & 17 & PLEKHM1 & $43,540,690$ & rs62055516 \\
\hline ENSG00000214425.2 & 17 & $\angle R R C 37 A 4 P$ & $43,603,193$ & rs389978 \\
\hline ENSG00000266918.1 & 17 & RP11-798G7.8 & $43,610,073$ & rs2668668 \\
\hline ENSG00000264070.1 & 17 & DND1P1 & $43,663,766$ & rs2266497 \\
\hline ENSG00000263503.1 & 17 & RP11-707O23.5 & $43,678,970$ & rs2266497 \\
\hline ENSG00000204650.9 & 17 & CRHR1-IT1 & $43,711,638$ & rs62061802 \\
\hline ENSG00000264589.1 & 17 & MAPT-ASI & $43,946,991$ & rs2668668 \\
\hline ENSG00000120071.8 & 17 & KANSL1 & $44,205,007$ & rs62073098 \\
\hline ENSG00000214401.4 & 17 & KANSL1-AS1 & $44,272,515$ & rs55974014 \\
\hline ENSG00000262539.1 & 17 & $R P 11-259 G 18.3$ & $44,337,444$ & rs56364632 \\
\hline ENSG00000176681.10 & 17 & $\angle R R C 37 A$ & $44,392,629$ & rs17660294 \\
\hline ENSG00000238083.3 & 17 & $L R R C 37 A 2$ & $44,609,846$ & rs439558 \\
\hline ENSG00000185829.11 & 17 & ARL17A & $44,625,578$ & rs3865315 \\
\hline ENSG00000120093.7 & 17 & HOXB3 & $46,646,933$ & rs890432 \\
\hline ENSG00000184009.5 & 17 & ACTG1 & $79,483,935$ & rs6565586 \\
\hline
\end{tabular}




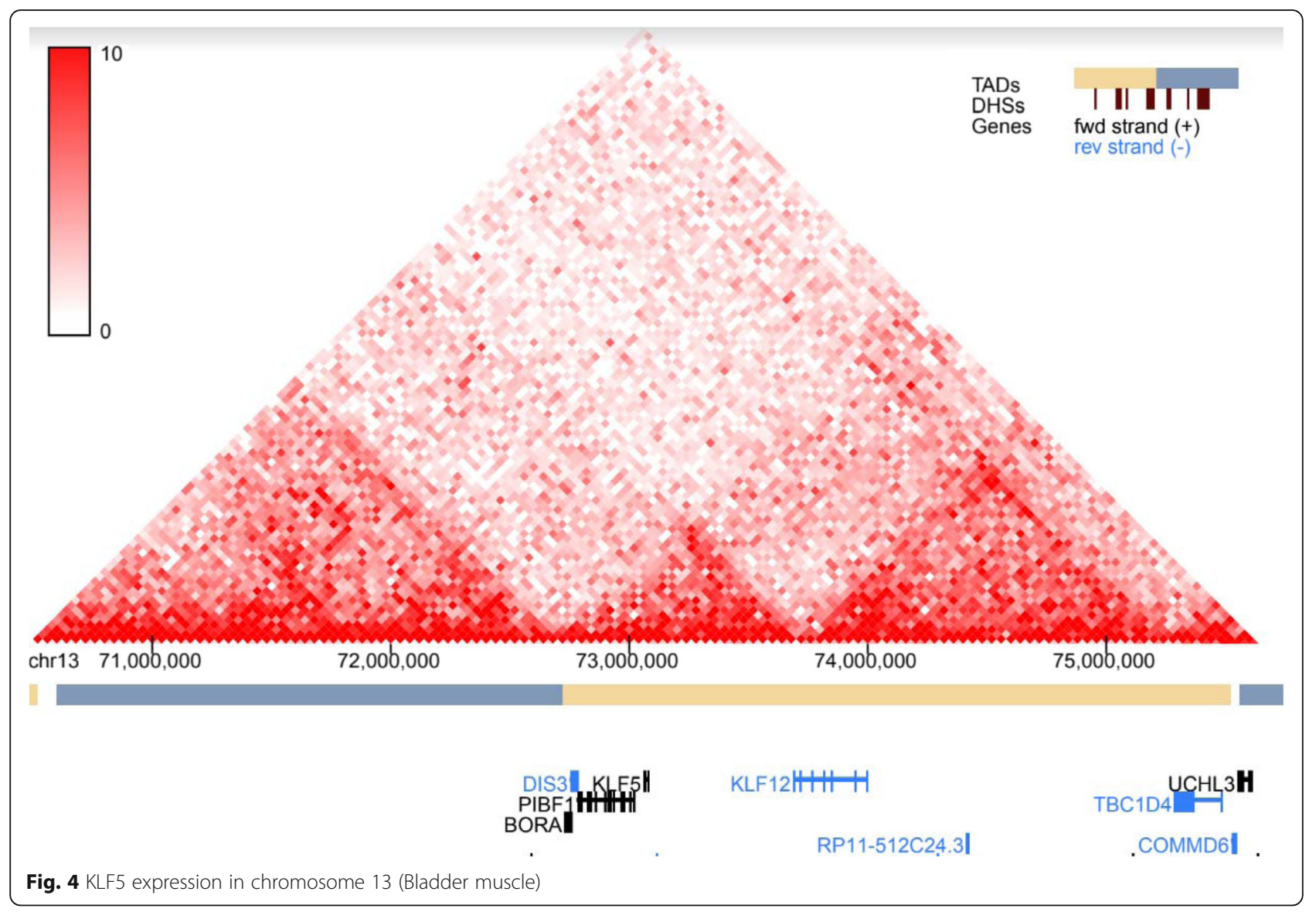

specific populations [20]. KLF5 (Kruppel Like Factor 5), encodes a member of the Kruppel-like factor subfamily of zinc finger proteins. The encoded protein is a transcriptional activator that binds directly to a specific recognition motif in the promoters of target genes. This protein acts downstream of multiple different signaling pathways and is regulated by post-translational modification [21]. GWAS catalogue doesn't relate this gene to muscle health phenotypes [19], the same is true for the Malacards [20] database. In contrast, the STRING database [22] finds relation between NUDT3 and ACTA2 (Actin Alpha 2) and GSK3B (Glycogen Synthase Kinase 3 Beta) which are related to actin production and energy metabolism respectively [21]. HLA-DQB1-AS1 (HLA$D Q B 1$ Antisense RNA 1) is an RNA Gene and is affiliated with the lncRNA (Long non-coding RNA) class is related to malignant diseases and doesn't seem to be associated with muscle wasting disorders according to MalaCards [20]. The above information emphasizes that these genes are not directly related to muscle health, yet they may have some indirect regulatory role in defining it. Functional validation is vital in the process of confirming or debunking the hypothesis that these genes are associated with muscle health. We suggest that the above genes be scrutinized using a scoring system as described below, for prioritizing candidate genes for functional validation which will be done by knocking out the gene in $\mathrm{C} 2 \mathrm{C} 12$ mouse myoblast cell, assessing gene expression using RT-qPCR and comparing cell morphology to the morphology wild type $\mathrm{C} 2 \mathrm{C} 12$ cells. The scoring system has been briefly stated in the form of abstract [23] earlier and we mention it in detail here. The following constitutes the scoring system proposed for functional validation of our results: Potential genes were obtained from the work of Zillikens et al. [8], Karasik et al. [7] for LM and for HG, Willems et al. [9] and Tikkanen et al. [10]. Genes provided by Karasik et al. Zillikens et al. and Willems et al. were graded as first tier genes, while genes provided by Tikkanen et al. were graded as second tier genes. The reason behind this is that the Tikkanen et al. research was published at the end of the year 2018, while the database was already being collected. The list of SNPs was mined with cis Expression quantitative trait loci analysis (eQTLs) for transcripts within $2 \mathrm{Mb}$ of the SNP position was carried out as described by Zillikens et al. [8]. Other similar 


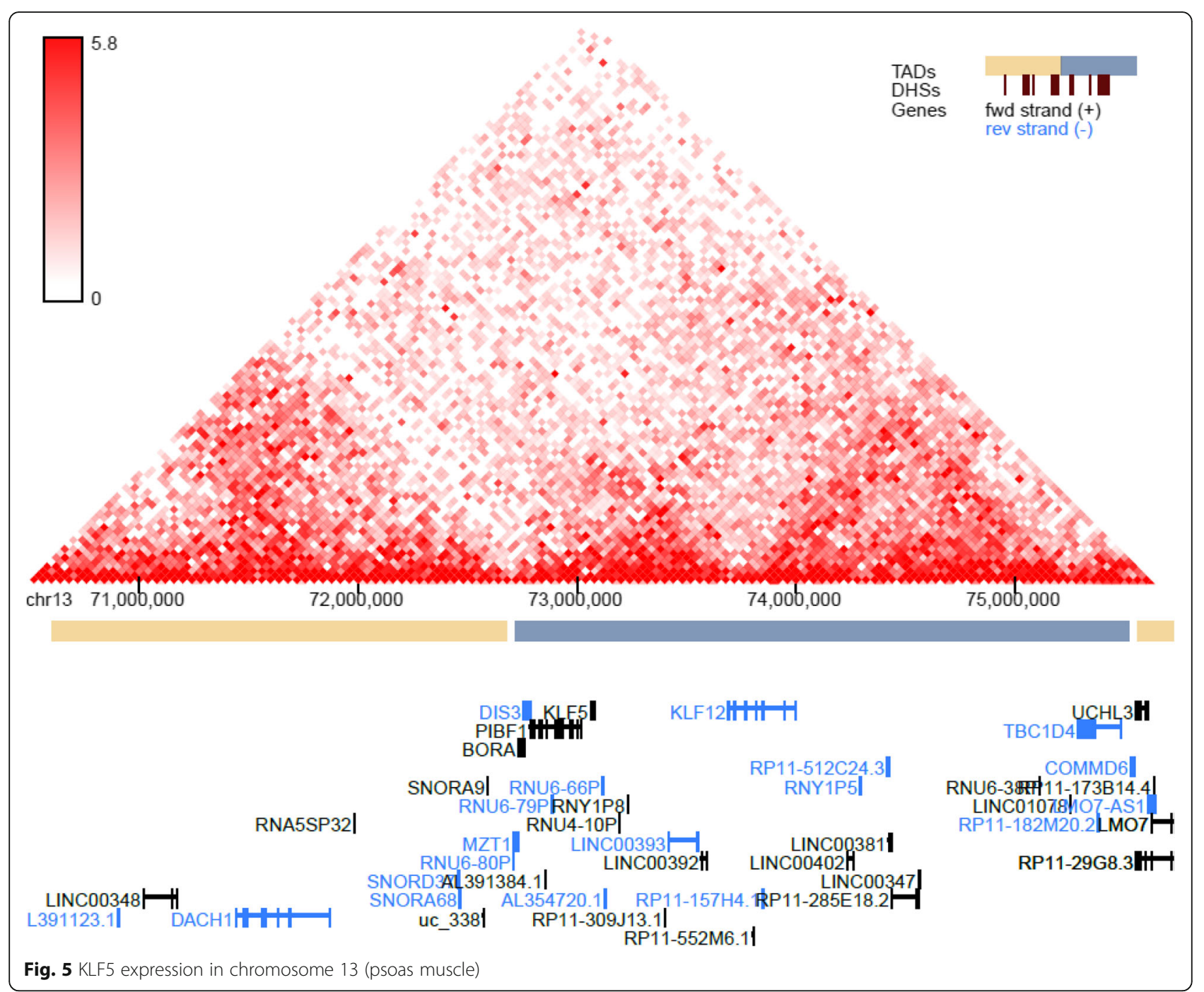

datasets were scrutinized, and genes in proximity of SNPs were scaled according to a specifically developed scoring system which utilized the following publicly available databases: Malacards [20], COXPRESdb [24] gene co-expression database, PubMed search engine, Ensembl database [25], the mouse genome informatics database [26], HaploReg [27] and the LDlink [28] database.

The above functional validation method combined with our approach to gene prioritization might help in Identifying new loci responsible for LM or HG, and thus identifying new genetic markers for sarcopenia. This approach may also be used by the pharmaceutical industry to identify targets for new pharmaceutical products or reposition existing drugs in accordance to new data on the activity of these drugs. The greatest hurdle with drug repositioning today is lack of solid databases needed to produce good results [24]. Functional validation of the results presented in this study results can serve as a test to whether our approach to gene prioritization can resolve this problem.

\section{Conclusions}

The current work focused primarily on the combined bioinformatic approaches using GWAS and eQTLs for SMR. The results of exclusivity of the tissues of interest were further classified for their importance based on Venn diagrams and their corresponding TAD plots to look for the TAD boundaries where the associated regulating SNPs could be localized. NUDT3 and KLF5 for lean mass and HLA-DQB1-AS1 for hand grip strength and their associated SNPs (rs464553, rs1028883 and rs3129753) had the highest priority as candidate targets for further study.

One limitation of this study is that the eQTL analysis was not done on trans-association SNPs. Another is the limited knowledge on TAD function.

We propose functional validation by a method of gene knock out in $\mathrm{C} 2 \mathrm{C} 12$ mouse myoblast cells to either 


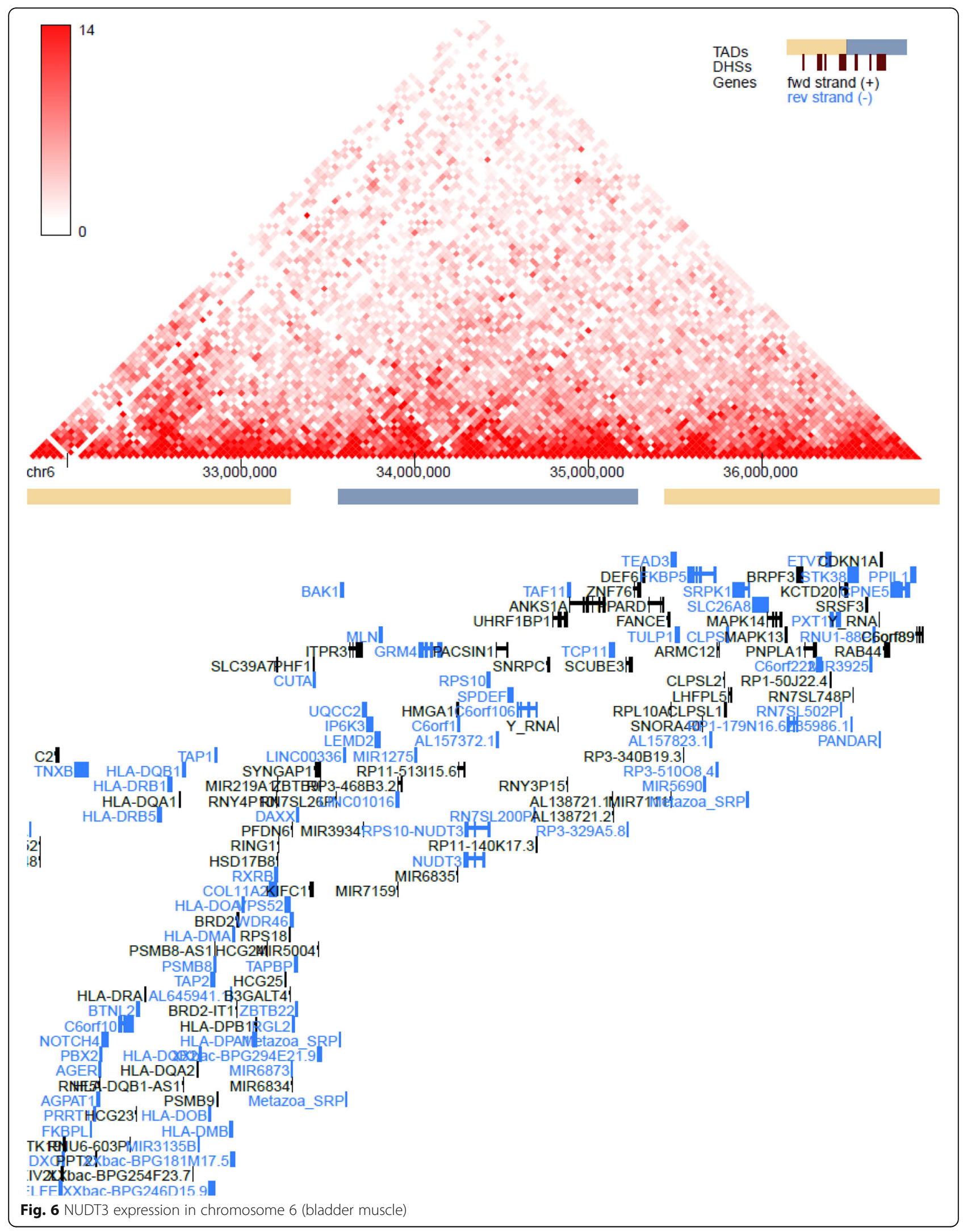




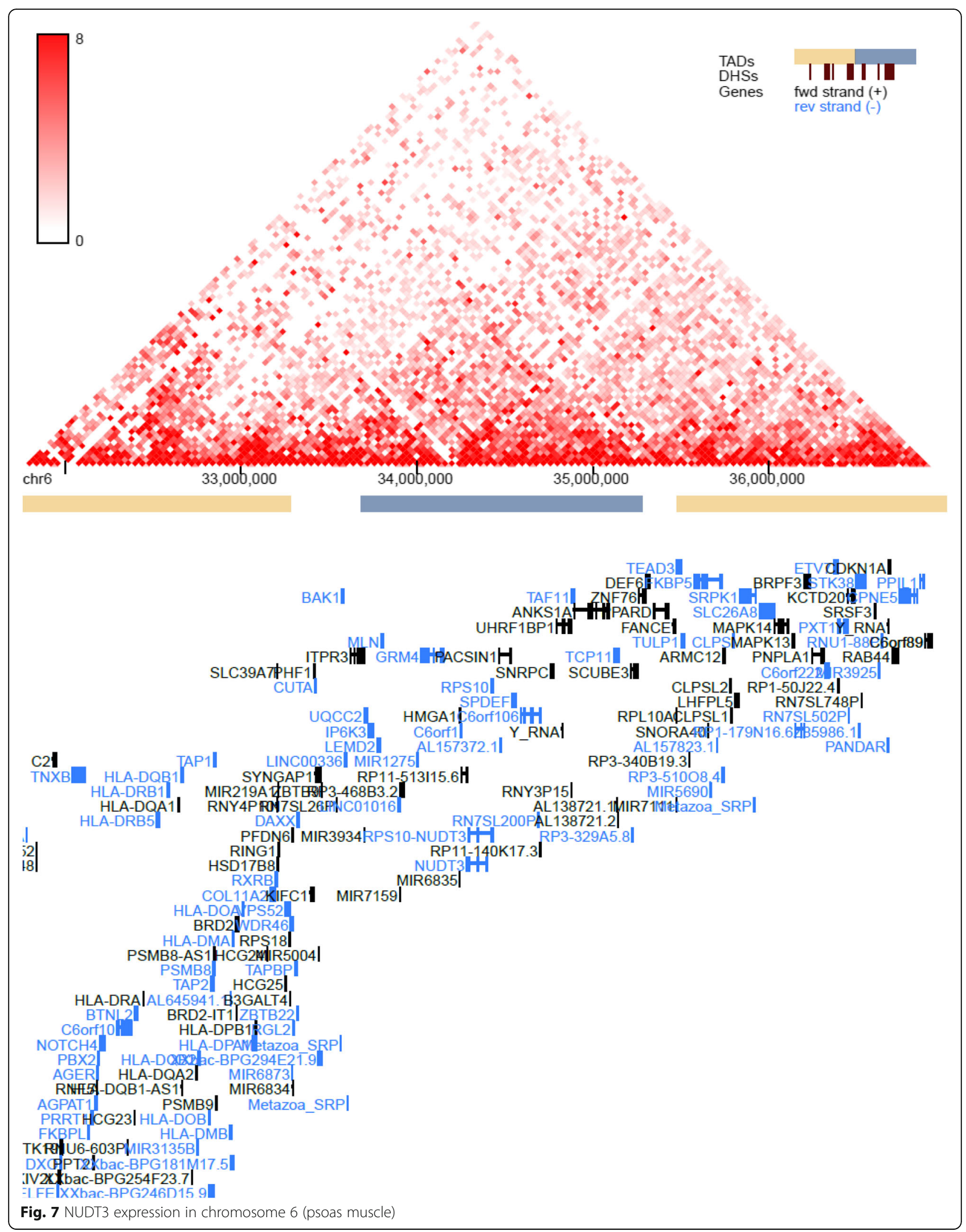


prove or rebut the effect of prioritized genes on muscle health, thus widening the scope of knowledge on the genetic origins of sarcopenia.

\section{Abbreviations}

eQTL: Expression quantitative trait loci; FIRE: Frequently interacting region; GTEx: Genotype Tissue Expression; GWAS: Genome wide association study; HG: Hand Grip strength; InDels: Insertions deletions; LD: Linkage Disequilibrium; LM: Lean Mass; IncRNA: Long non-coding RNA; Metal: Metaanalysis; RT-qPCR: Real Time quantitative polymerase chain reaction; SMR: Summary-data-based Mendelian randomization; SNPs: Single Nucleotide Polymorphism; TADs: Topologically Associated Domains: TWAS: Transcriptome Wide Association Study

\section{Acknowledgements}

The authors are thankful for the various software tools that have been made available for academic purposes such as SMR tool. Authors are also thankful to David Karasik of Bar Ilan University who provided GWAS data that he carried out for hand grip and lean mass study for his previous research work David Laaksonen of UEF helped in contribution of the written content of the manuscript.

\section{Authors' contributions}

ANS conceived the presented idea, performed the statistical computational analysis of data and worked on the manuscript. BG provided the functional validation algorithm, designed the scoring system and contributed to the manuscript. The author(s) read and approved the final manuscript.

\section{Funding}

This research was funded by the: "ISRAEL SCIENCE FOUNDATION (grant No. $1121 / 19)^{\prime \prime}$

\section{Availability of data and materials}

www.tinyurl.com/abinarain and then navigate to Educational Section and click where its written 'GWAS eQTL Summary Approach with TADs for Skeletal Muscle work'.

\section{Ethics approval and consent to participate}

Not applicable.

\section{Consent for publication}

Not applicable.

\section{Competing interests}

The authors declare that they have no competing interests.

Received: 16 September 2019 Accepted: 18 February 2020

Published online: 24 February 2020

\section{References}

1. Cruz-Jentoft AJ, Baeyens JP, Bauer JM, Boirie Y, Cederholm T, Landi F, et al. Sarcopenia: European consensus on definition and diagnosis. Age Ageing. 2010;39(4):412-23.

2. Haines JL, Hauser MA, Schmidt S, Scott WK, Olson LM, Gallins P, et al. Complement factor $\mathrm{H}$ variant increases the risk of age-related macular degeneration. Science (80- ), 2005. 308(5720):419-21.

3. Barbe MF, Gallagher S, Massicotte VS, Tytell M, Popoff SN, Barr-Gillespie AE. The interaction of force and repetition on musculoskeletal and neural tissue responses and sensorimotor behavior in a rat model of work-related musculoskeletal disorders. BMC Musculoskelet Disord [Internet]. 2013;14(1): 303 Available from: http://bmcmusculoskeletdisord.biomedcentral.com/ articles/10.1186/1471-2474-14-303. [cited 2019 Jan 26].

4. Aguet F, Brown AA, Castel SE, Davis JR, He Y, Jo B, et al. Genetic effects on gene expression across human tissues. Nature. 2017;550(7675):204-13.

5. Westra HJ, Peters MJ, Esko T, Yaghootkar H, Schurmann C, Kettunen J, et al. Systematic identification of trans eQTLs as putative drivers of known disease associations. Nat Genet. 2013:45(10):1238-43.

6. Pombo A, Dillon N. Three-dimensional genome architecture: Players and mechanisms. Vol. 16, Nature Reviews Molecular Cell Biology. Nat Publ Group. 2015:245-57.
7. Karasik D, Zillikens MC, Hsu YH, Aghdassi A, Akesson K, Amin N, et al. Disentangling the genetics of lean mass. Am J Clin Nutr. 2019:109(2):276-8.

8. Zillikens MC, Demissie S, Hsu Y-H, Yerges-Armstrong LM, Chou W-C, Stolk L, et al. Large meta-analysis of genome-wide association studies identifies five loci for lean body mass. Nat Commun [Internet]. 2017;8(1):80 Available from: http://www.nature.com/articles/s41467-017-00031-7. [cited 2019 Feb 11].

9. Willems SM, Wright DJ, Day FR, Trajanoska K, Joshi PK, Morris JA, et al. Large-scale GWAS identifies multiple loci for hand grip strength providing biological insights into muscular fitness. Nat Commun [Internet]. 2017;8: 16015 Available from: http://www.nature.com/doifinder/10.1038/ ncomms16015. [cited 2019 Feb 11].

10. Tikkanen E, Gustafsson S, Amar D, Shcherbina A, Waggott D, Ashley EA, et al. Biological Insights Into Muscular Strength: Genetic Findings in the UK Biobank. Sci Rep [Internet]. 2018;8(1):6451 Available from: http://www. nature.com/articles/s41598-018-24735-y. [cited 2019 Feb 11].

11. Zhu Z, Zhang F, Hu H, Bakshi A, Robinson MR, Powell JE, et al. Integration of summary data from GWAS and eQTL studies predicts complex trait gene targets. Nat Genet. 2016;48(5):481-7.

12. Schmitt AD, Hu M, Jung I, Xu Z, Qiu Y, Tan CL, et al. A Compendium of Chromatin Contact Maps Reveal Spatially Active Regions in the Human Genome HHS Public Access. Cell Rep [Internet]. 2016;17(8):2042-59 Available from: www.cell.com/. [cited 2019 Dec 21].

13. Dixon JR, Selvaraj S, Yue F, Kim A, Li Y, Shen Y, et al. Topological Domains in Mammalian Genomes Identified by Analysis of Chromatin Interactions HHS Public Access. Nature [Internet]. 485(7398):376-80 Available from: http:// www.nature.com/authors/editorial_policies/license.html\#termshttp:// chromosome.sdsc.edu/mouse/hi-c/database.html. [cited 2019 Dec 21].

14. Lupiáñez DG, Kraft K, Heinrich V, Krawitz P, Brancati F, Klopocki E, et al. Disruptions of topological chromatin domains cause pathogenic rewiring of gene-enhancer interactions HHS public access. Cell. 2015;161(5):1012-25.

15. Narendra V, Rocha PP, An D, Raviram R, Skok JA, Mazzoni EO, et al CTCF establishes discrete functional chromatin domains at the Hox clusters during differentiation. Available from: http://www.ncbi.nlm.nih.gov/geo/ [cited 2019 Dec 21]

16. Flavahan WA, Drier Y, Liau BB, Gillespie SM, Venteicher AS, StemmerRachamimov AO, et al. Insulator dysfunction and oncogene activation in IDH mutant gliomas HHS Public Access. Nature [Internet]. 2016;529(7584): 110-4 Available from: http://www.nature.com/authors/editorial_policies/ license.html\#terms. [cited 2019 Dec 21].

17. Doostparast Torshizi A, Wang K. Next-generation sequencing in drug development: target identification and genetically stratified clinical trials. Vol. 23, Drug Discovery Today. Elsevier Ltd. 2018:1776-83.

18. Safrany ST. A novel context for the 'MutT' module, a guardian of cell integrity, in a diphosphoinositol polyphosphate phosphohydrolase. EMBO J. 1998:17(22):6599-607.

19. Buniello A, Macarthur JAL, Cerezo M, Harris LW, Hayhurst J, Malangone C, et al. The NHGRI-EBI GWAS catalog of published genome-wide association studies, targeted arrays and summary statistics 2019. Nucleic Acids Res. 2018:47:1005-12.

20. Rappaport N, Twik M, Plaschkes I, Nudel R, Stein TI, Levitt J, et al. MalaCards: an amalgamated human disease compendium with diverse clinical and genetic annotation and structured search. Nucleic Acids Res [Internet]. 2017; 45:877-87 Available from: https://academic.oup.com/nar/article-abstract/45/ D1/D877/2572056. [cited 2019 Feb 11].

21. Stelzer G, Rosen N, Plaschkes I, Zimmerman S, Twik M, Fishilevich S, et al. The GeneCards Suite: From Gene Data Mining to Disease Genome Sequence Analyses. Curr Protoc Bioinforma [Internet]. 2016;54(1) Available from: https://onlinelibrary.wiley.com/doi/abs/https://doi.org/10.1002/cpbi.5. [cited 2019 Dec 21].

22. Szklarczyk D, Gable AL, Lyon D, Junge A, Wyder S, Huerta-Cepas J, et al. STRING V11: protein-protein association networks with increased coverage, supporting functional discovery in genome-wide experimental datasets. Nucleic Acids Res [Internet]. 2018;47:607-13 Available from: https://stringdb.org/. [cited 2019 Dec 22].

23. Gasman B, Baum G, Karasik D. C2C12 myoblast gene knockout to validate the findings of genome-wide association study of muscle traits, Journal of Frailty \& Aging, Forthcoming 2020

24. Obayashi T, Hayashi S, Shibaoka M, Saeki M, Ohta H, Kinoshita K. COXPRESdb: a database of coexpressed gene networks in mammals. Nucleic Acids Res [Internet]. 2008;36:77-82 Available from: http://coxpresdb. [cited 2019 Dec 21]. 
25. Zerbino DR, Achuthan P, Akanni W, Ridwan Amode M, Barrell D, Bhai J, et al. Ensembl 2018. Nucleic Acids Res [Internet]. 2018;46 Available from: http://www.ensembl.org.[cited 2019 Feb 11].

26. Finger JH, Smith CM, Hayamizu TF, Mccright IJ, Xu J, Law M, et al. The mouse Gene Expression Database (GXD): 2017 update. Nucleic Acids Res [Internet]. 2017;45 Available from: http://www.informatics.jax.org/gxdlit. [cited 2019 Dec 21].

27. Ward LD, Kellis M. HaploReg: A resource for exploring chromatin states, conservation, and regulatory motif alterations within sets of genetically linked variants. Nucleic Acids Res [Internet]. 2012;40(D1):930-4 Available from: http://compbio.mit.edu/HaploReg. [cited 2019 Apr 22].

28. Machiela MJ, Chanock SJ. Genetics and population analysis LDlink: a webbased application for exploring population-specific haplotype structure and linking correlated alleles of possible functional variants.; Available from: https:/academic.oup.com/bioinformatics/article-abstract/31/21/3555/195027. [cited 2019 Mar 21]

\section{Publisher's Note}

Springer Nature remains neutral with regard to jurisdictional claims in published maps and institutional affiliations.

Ready to submit your research? Choose BMC and benefit from:

- fast, convenient online submission

- thorough peer review by experienced researchers in your field

- rapid publication on acceptance

- support for research data, including large and complex data types

- gold Open Access which fosters wider collaboration and increased citations

- maximum visibility for your research: over $100 \mathrm{M}$ website views per year

At BMC, research is always in progress.

Learn more biomedcentral.com/submissions 